\title{
Staphylococcus massiliensis isolated from human blood cultures, Germany, 2017-2020
}

\author{
Katharina Last ${ }^{1} \cdot$ Philipp M. Lepper ${ }^{2} \cdot$ Philipp Jung $^{1} \cdot$ Hans-Joachim Schäfers ${ }^{3} \cdot$ Sébastien Boutin $^{4} \cdot$ Klaus Heeg $^{4}$. \\ Sören L. Becker ${ }^{1} \cdot$ Dennis Nurjadi $^{4}$. Cihan Papan ${ }^{1} \mathbb{C}$
}

Received: 9 August 2021 / Accepted: 18 January 2022 / Published online: 26 January 2022

(c) The Author(s) 2022

\begin{abstract}
Clinical and laboratory data on newly described staphylococcal species is rare, which hampers decision-making when such pathogens are detected in clinical specimens. Here, we describe Staphylococcus massiliensis detected in three patients at a university hospital in southwest Germany. We report the discrepancy of microbiological findings between matrix-assisted laser desorption/ionization time-of-flight mass spectrometry, 16S-rRNA polymerase chain reaction, and whole-genome sequencing for all three isolates. Our findings highlight the diagnostic pitfalls pertinent to novel and non-model organisms in daily microbiological practice, in whom the correct identification is dependent on database accuracy.
\end{abstract}

Keywords Coagulase-negative staphylococci $\cdot$ Staphylococcus massiliensis $\cdot$ Non-model organisms $\cdot$ Novel species · Whole-genome sequencing $\cdot 16 \mathrm{~S}$ rRNA $\cdot$ MALDI-TOF MS

\section{Introduction}

Coagulase-negative staphylococci (CoNS) are abundant inhabitants of the human skin and the mucosa with limited pathogenicity [1, 2], as opposed to Staphylococcus aureus [3] and other members of the S. aureus complex [4, 5]. Device-related infections are one of the most common manifestations of CoNS infections, causing high morbidity and mortality [6, 7]. With the advent of modern tools in routine microbiological diagnostics such as matrix-assisted laser desorption/ionization time-of-flight mass spectrometry

Dennis Nurjadi and Cihan Papan are senior authors

Cihan Papan

cihan.papan@uks.eu

1 Center for Infectious Diseases, Institute of Medical Microbiology and Hygiene, Saarland University, Kirrberger Strasse, Building 43, 66421 Homburg, Germany

2 Department of Pneumology, Allergology and Critical Care Medicine, Saarland University, Homburg, Germany

3 Department of Thoracic and Cardiovascular Surgery, Saarland University, Homburg, Germany

4 Department of Infectious Diseases, Medical Microbiology and Hospital Hygiene, University Hospital Heidelberg, Heidelberg, Germany
(MALDI-TOF MS), pathogen detection has experienced a remarkable refinement within the past decade [8, 9]. However, reliable species identification by MALDI-TOF MS relies greatly on the quality and accuracy of the employed database. Over the past years, the species identification and differentiation of clinical CoNS isolates have improved significantly, leading to the identification of novel or "rare" species in clinical specimens [10]. Indeed, the "true" clinical relevance of many CoNS species is frequently underappreciated. Staphylococcus massiliensis is a recently described CoNS first reported in 2010 from a cerebral abscess [11], and later from healthy human skin [12]. In 2012, Zong postulated to conceptualize $S$. massiliensis as part of the normal human skin microflora [13]. Until today, data pertaining to the pathogenic potential of this microorganism and possible treatment approaches is scarce. Here, we report the challenges and limitations in the identification of $S$. massiliensis in blood cultures from three patients at a university hospital in southwest Germany. 


\section{Methods}

\section{Microbiological characterization and MALDI-TOF MS species identification}

We performed a retrospective search of our microbiological database to identify $S$. massiliensis isolates in blood cultures from January 2017 to December 2020. We screened the database for blood culture isolates deposited as "coagulase-negative staphylococci," suggesting that a definitive identification with MALDI-TOF MS failed in these isolates. All samples were processed in accordance with standard microbiological procedures, as described previously [4]. For isolate identification, a combination of MALDI-TOF MS (Biotyper ${ }^{\mathrm{TM}}$; Bruker Daltonics, Bremen, Germany, with the MBT Compass 4.1 database containing 7588 bacterial pathogens but not including $S$. massiliensis) according to the manufacturer's protocols and sequencing of the 16S-rRNA coding gene was utilized (see below). Antimicrobial susceptibility testing (AST) was carried out on a VITEK II (BioMérieux, Marcy l'Étoile, France) and interpreted in accordance with the guidelines of the European Committee on Antimicrobial Susceptibility Testing (EUCAST, version 10.0). Data on the number of positive blood culture bottles and the time to positivity was collected.

\section{Sequencing of the $16 \mathrm{~S}$ rRNA coding gene}

A fragment of the 16S-rRNA coding gene was amplified, using the primer sequences (5'-3') AGAGTTTGATCMTGGCTCAG (forward) and CCGTCAATTCMTTTGAG TTT (reverse), respectively. Subsequently, the PCR product was processed with the AMPure PCR purification kit (BeckmanCoulter, Krefeld, Germany) and dye-labeled using the DTCS Quick Start Kit (Sciex, Framingham, USA) with the primer sequences (5'-3') AGAGTTTGATCMTGGCTC AG (forward) and GWATTACCGCGGCKGCTG (reverse), respectively. The dye-labeled products were purified with the CleanSEQ Dye-Terminator Removal Kit (BeckmanCoulter, Krefeld, Germany) and sequenced via capillary electrophoresis (GenomeLab GeXP; BeckmanCoulter, Krefeld, Germany). The forward and reverse strands were processed and analyzed individually. Sequencing and all kit reactions were performed according to the manufacturer's protocols. The PCR protocols are displayed in Table 1.

\section{Whole-genome sequencing}

DNA extraction and short-read Illumina sequencing were performed as previously described [14]. For correct species designation, core genome alignment was performed
Table 1 PCR protocols for amplification of the 16S rRNA coding gene and dye-labeling prior DNA sequencing

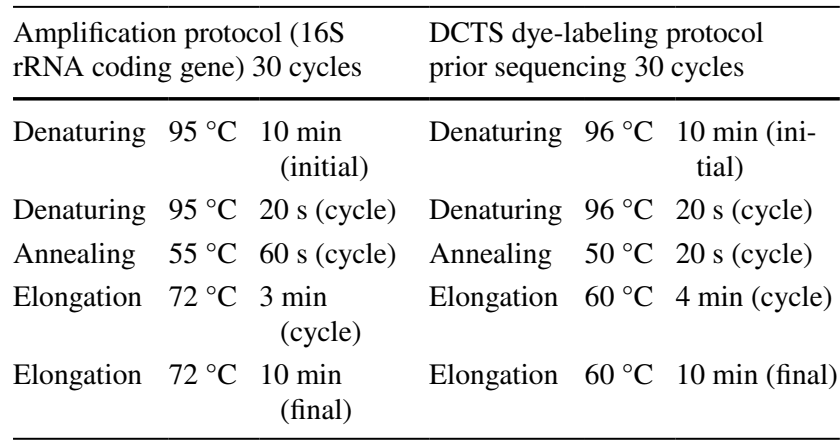

with publicly available Staphylococcus haemolyticus and Staphylococcus saprophyticus genomes, which showed the closest phylogenetic sequence homologies. The sequences were deposited at the NCBI GenBank under the Bioproject PRJNA750592.

\section{Patient characteristics}

Review of the patient charts was performed to extract clinical data, including basic demographics, comorbidities, concomitant infectious syndromes and pathogens detected, presence of foreign bodies, and the antibiotic treatment.

\section{Ethics}

This study was approved by the local ethics committee (Number 147/21). Due to the retrospective nature of the study and the anonymization of patient data, the need for informed consents was waived.

\section{Results}

\section{Microbiological characterization}

Between January 2017 and December 2020, a total of 203 isolates were found to be deposited as "coagulase-negative staphylococci" in our microbiological database. Of these, 181 were in a second step identified per MALDI-TOF MS as Staphylococcus epidermidis or other, common species, and in 13 cases, a polymicrobial culture was found and/or the initial suspicion of CoNS was refuted. In the remaining nine cases, MALDI-TOF MS yielded either Staphylococcus sp. or no reliable identification. Out of this cohort of nine isolates, four blood culture bottles from three different patients were positive for $S$. massiliensis. The clinical characteristics are provided in Table 2. MALDI-TOF MS yielded no reliable identification of all three isolates (all scores $\leq 1.47$, for detailed information refer to Table 3 ), 
Table 2 Characteristics of patients with positive blood cultures for S. massiliensis, treated at the Saarland University, Germany, 2017 to 2020

\begin{tabular}{|c|c|c|c|}
\hline & Patient \#1 (P1) & Patient \#2 (P2) & Patient \#3 (P3) \\
\hline Age at presentation & 67 & 79 & 53 \\
\hline Sex & Male & Female & Male \\
\hline $\begin{array}{l}\text { Number of positive blood culture bottle } \\
\text { sets, in relation to total number of } \\
\text { blood cultures drawn }\end{array}$ & 1/1 (peripheral) & 1/1 (peripheral) & $1 / 2$ (peripheral) \\
\hline Positive bottle type(s) & Aerobic & Aerobic & Aerobic and anaerobic \\
\hline $\begin{array}{l}\text { Time to positivity of blood culture } \\
\text { (hours) }\end{array}$ & 28 & 73 & 18 \\
\hline Clinical syndrome & Aortic insufficiency, aortitis & Fever without source & COVID-19 \\
\hline Other pathogens recovered & $\begin{array}{l}\text { Staphylococcus epidermidis, per culture } \\
\text { (aortic valve) } \\
\text { Staphylococcus aureus, per PCR (pSA- } \\
\text { 442) (aortic valve) }\end{array}$ & $\begin{array}{l}\text { Escherichia coli (urine), Citrobac- } \\
\text { ter freundii (urine) }\end{array}$ & - \\
\hline Antibiotic treatment & $\begin{array}{l}\text { Daptomycin; later switched to vanco- } \\
\text { mycin + rifampicin }\end{array}$ & Ampicillin/sulbactam; meropenem & $\begin{array}{l}\text { Azithromycin; pipera- } \\
\text { cillin/tazobactam }\end{array}$ \\
\hline Foreign body & Aortic valve and root replacement & Total knee replacement & Central venous catheter \\
\hline Comorbidities & See above & Lung adenocarcinoma & COVID-19 \\
\hline
\end{tabular}

which were subsequently diagnosed as $S$. massiliensis via sequencing of the 16S-rRNA coding gene and further investigated by whole-genome sequencing (WGS). All isolates were methicillin-susceptible (Table 4).

\section{Sequence analyses of the 16S-rRNA coding gene}

PCR of isolate P1 (of patient \#1) yielded a coverage of $100 \%$ each on the forward and reverse strands (671 and 465 base pairs), with $99.85 \%$ and $99.57 \%$ identification of $S$. massiliensis, respectively. Isolate P2 was diagnosed as $S$. massiliensis with coverage of $100 \%$ for both strands (forward 414 base pairs, reverse 386 base pairs), while identification resulted in $99.03 \%$ and $97.2 \%$, respectively. Isolate P3 (forward 718 base pairs, reverse 424 base pairs) resulted in $100 \%$ coverage each, and an identification of $99.72 \%$ and $100 \%$, respectively, as S. massiliensis.

\section{WGS}

The draft genome was analyzed using KmerFinder and two different species were suggested as best hit: S. haemolyticus and S. saprophyticus. S. massiliensis is not included in the database of KmerFinder; therefore, we compared our sequences to the 3 publicly available genomes of $S$. massiliensis as well as the complete genome publicly available in the refseq database. The core genome used for the comparison was relatively small (27 genes, 1661 polymorphic sites) but revealed a close phylogentic relationship between our isolates and the 3 genomes belonging to $S$. massiliensis (Fig. 1A; Table 5). To validate these results, we refined the core genome (1987 genes, 3093 polymorphic sites) using only the common genes among those 6 isolates. This analysis validated the genetic identity of our isolate as $S$. massiliensis with a higher confidence. These results are also in line with the average nucleotide identity (ANI) of $99.29 \%$ (99.19-99.38\%) between our 3 isolates and the 3 available genomes of $S$. massiliensis, with an ANI value $>95 \%$ being a reliable indicator for species identity [15]. Although our analyzed isolates all belonged to $S$. massiliensis, they showed a different resistome pattern (Fig. 1B). We found a high level of resistance with 6 resistance genes in isolate P3, while the reference genomes were overall more susceptible to antibiotics.

\section{Discussion}

We describe here the incongruency of bacterial species identification for S. massiliensis using three different methods, namely sequencing of the $16 \mathrm{~S}$ rRNA coding gene, MALDITOF MS, and WGS. While Sanger sequencing of the $16 \mathrm{~S}$ rRNA coding gene correctly identified $S$. massiliensis, both mass spectrometry and next-generation sequencing were unable to clearly distinguish $S$. massiliensis from closely related staphylococcal species. Indeed, Zong previously reported the misidentification as Staphylococcus simulans by the MicroScan Walkaway 96 system [13]. Our results demonstrate that even modern and high-resolution identification techniques, such as MALDI-TOF MS and WGS, are dependent on the quality and currentness of the database. Although WGS is frequently regarded as a new gold standard for the molecular characterization of bacteria, the used databases are not necessarily reliable for newly described 
Table 3 MALDI-TOF MS identification (ten highest scores) with ambiguous results, which were subsequently identified as $S$. massiliensis via sequencing of the $16 \mathrm{~S}$ rRNA coding gene

\begin{tabular}{|c|c|c|}
\hline Species & MALDI-TOF MS score & NCBI code \\
\hline \multicolumn{3}{|l|}{$\mathrm{P} 1$} \\
\hline Staphylococcus cohnii spp. cohnii & 1.38 & 74704 \\
\hline Staphylococcus cohnii spp. cohnii & 1.37 & 74704 \\
\hline Staphylococcus equorum spp. equorum & 1.37 & 246432 \\
\hline Staphylococcus hominis & 1.35 & 1290 \\
\hline Staphylococcus pasteuri & 1.34 & 45,972 \\
\hline Staphylococcus haemolyticus & 1.34 & 1283 \\
\hline Staphylococcus warneri & 1.31 & 1292 \\
\hline Staphylococcus hominis spp. novobiosepticus & 1.31 & 145393 \\
\hline Staphylococcus xylosus & 1.30 & 1288 \\
\hline Lactobacillus mисоsae & 1.29 & 97478 \\
\hline \multicolumn{3}{|l|}{$\mathrm{P} 2$} \\
\hline Staphylococcus xylosus & 1.47 & 1288 \\
\hline Staphylococcus equorum spp. equorum & 1.45 & 246432 \\
\hline Staphylococcus cohnii spp. cohnii & 1.36 & 74704 \\
\hline Staphylococcus saprophyticus spp. saprophyticus & 1.35 & 147452 \\
\hline Staphylococcus cohnii spp. cohnii & 1.35 & 74704 \\
\hline Staphylococcus xylosus & 1.30 & 1288 \\
\hline Staphylococcus carnosus spp. utilis & 1.30 & 147,449 \\
\hline Arthrobacter pascens & 1.29 & 1677 \\
\hline Staphylococcus carnosus spp. carnosus & 1.28 & 147448 \\
\hline Staphylococcus cohnii spp. urealyticus & 1.28 & 94138 \\
\hline \multicolumn{3}{|l|}{$\mathrm{P} 3$} \\
\hline Staphylococcus carnosus spp. utilis & 1.45 & 147449 \\
\hline Staphylococcus chromogenes & 1.44 & 46,126 \\
\hline Staphylococcus lugdunensis & 1.39 & 28035 \\
\hline Staphylococcus equorum spp. equorum & 1.35 & 246432 \\
\hline Staphylococcus xylosus & 1.30 & 1288 \\
\hline Staphylococcus xylosus & 1.30 & 1288 \\
\hline Staphylococcus felis & 1.29 & 46127 \\
\hline Staphylococcus xylosus & 1.28 & 1288 \\
\hline Lactobacillus oligofermentas & 1.28 & 293371 \\
\hline Staphylococcus warneri & 1.27 & 1292 \\
\hline
\end{tabular}

species and non-model organisms. The clinical relevance of $S$. massiliensis is still unclear. In all three patients, we detected S. massiliensis in the blood culture. Despite clear clinical signs of infection and inflammatory processes, the interpretation of blood culture positivity for $S$. massiliensis remains difficult. In both, patients 1 and 2, other clinically relevant pathogens were detected, and COVID-19 was diagnosed in patient 3 , thus rendering a causative role of $S$. massiliensis uncertain. In addition, only one blood culture pair was taken for patients 1 and 2, and the time to positivity for all blood culture bottles was rather long $(>16 \mathrm{~h}$ ) for all three patients, so that contamination cannot be completely ruled out. Thus, no definitive statement can be made regarding the clinical relevance of the $S$. massiliensis findings in our patients. Nonetheless, the accuracy of species identification is essential for further investigations on the clinical relevance of $S$. massiliensis as a potential pathogen. While the detection of CoNS in blood cultures is frequently considered contamination, specific CoNS species, such as Staphylococcus epidermidis and Staphylococcus capitis, may well be clinically relevant, in particular if indwelling devices are present [16]. The first description of S. massiliensis dates back to 2010, when Al Malsalma and colleagues reported the detection of this staphylococcal species from clinical specimen of a 52-year-old male patient (from 2005) with a brain tumor [11]. He developed a cerebral abscess after neurosurgery, which was drained and sent for microbiological diagnostics. Indeed, CoNS can cause post-surgical intracranial infections, rendering the findings of Al Malsalma et al. plausible. However, S. massiliensis has also been suggested as commensal of the human skin as it has been found in the skin of a fresh wound and healthy human skin $[12,13]$. On 
Table 4 Antibiotic susceptibility profile of $S$. massiliensis isolates detected in blood cultures at the Saarland University, Germany, 2017 to $2020(n=3)$

\begin{tabular}{|c|c|c|c|c|c|c|}
\hline \multirow[t]{2}{*}{ Antimicrobial substance } & \multicolumn{2}{|l|}{ P1 } & \multicolumn{2}{|l|}{$\mathrm{P} 2$} & \multicolumn{2}{|l|}{$\mathrm{P} 3$} \\
\hline & MIC & Int & MIC & Int & MIC & Int \\
\hline Flucloxacillin & $\leq 0.25$ & $\mathrm{~S}$ & $\leq 0.25$ & $\mathrm{~S}$ & $\leq 0.25$ & $\mathrm{~S}$ \\
\hline Gentamicin & $\leq 0.5$ & $\mathrm{~S}$ & $\leq 0.5$ & $\mathrm{~S}$ & $\leq 0.5$ & $\mathrm{~S}$ \\
\hline Ciprofloxacin & $\leq 0.5$ & I & $\leq 0.5$ & I & $\leq 0.5$ & I \\
\hline Moxifloxacin & $\leq 0.25$ & $\mathrm{~S}$ & $\leq 0.25$ & $\mathrm{~S}$ & $\leq 0.25$ & $\mathrm{~S}$ \\
\hline Erythromycin & $\leq 0.25$ & $\mathrm{~S}$ & 0.5 & $\mathrm{~S}$ & $\geq 8$ & $\mathrm{R}$ \\
\hline Clindamycin & $\leq 0.25$ & $\mathrm{~S}$ & $\leq 0.25$ & $\mathrm{~S}$ & $\leq 0.25$ & $\mathrm{~S}$ \\
\hline Linezolid & 1 & $\mathrm{~S}$ & 1 & $\mathrm{~S}$ & 2 & $\mathrm{~S}$ \\
\hline Daptomycin & 0.25 & $\mathrm{~S}$ & 0.25 & $\mathrm{~S}$ & 0.25 & $\mathrm{~S}$ \\
\hline Vancomycin & $\leq 0.5$ & $\mathrm{~S}$ & $\leq 0.5$ & $\mathrm{~S}$ & $\leq 0.5$ & $\mathrm{~S}$ \\
\hline Tetracycline & $\leq 1$ & $\mathrm{~S}$ & $\leq 1$ & $\mathrm{~S}$ & $\leq 1$ & $\mathrm{~S}$ \\
\hline Tigecycline & $\leq 1$ & $\mathrm{~S}$ & $\leq 0.12$ & $\mathrm{~S}$ & $\leq 0.12$ & $\mathrm{~S}$ \\
\hline Fosfomycin & $\leq 0.12$ & $\mathrm{R}$ & $\geq 128$ & $\mathrm{R}$ & $\leq 8$ & $\mathrm{~S}$ \\
\hline Fusidic acid & $\geq 128$ & $\mathrm{R}$ & 2 & $\mathrm{R}$ & $\leq 0.5$ & $\mathrm{~S}$ \\
\hline Rifampicin & $\leq 0.5$ & $\mathrm{~S}$ & $\leq 0.5$ & $\mathrm{~S}$ & $\leq 0.5$ & $\mathrm{~S}$ \\
\hline Co-trimoxazole & $\leq 10$ & $\mathrm{~S}$ & $\leq 10$ & $\mathrm{~S}$ & $\leq 10$ & $\mathrm{~S}$ \\
\hline
\end{tabular}

MICs are displayed as reported by VITEK2; antibiotic susceptibility was interpreted according to the EUCAST breakpoints v10.0. I, susceptible, increased exposure; Int, interpretation; $M I C$, minimal inhibitory concentration (in $\mathrm{mg} / \mathrm{L}$ ); $R$, resistant; $S$, susceptible

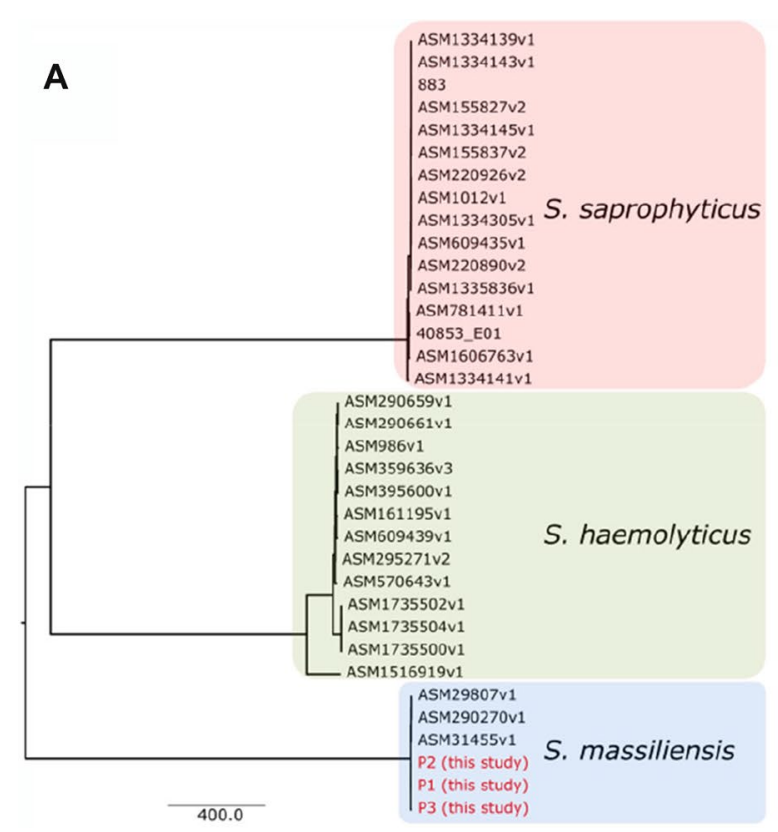

Fig. 1 Phylogenetic tree of Staphylococcus massiliensis isolates and closely related Staphylococcus species. A Genetic identification of our isolates (P1, P2, and $\mathrm{P} 3$ ) as $S$. massiliensis compared to publicly available genomes of closely related species ( $S$. saphrophyticus, $S$.

a different note, consistent with the findings of Zong et al. of the presence of a mobile staphylococcal cassette chromosome mec in S. massiliensis [17], we detected the presence of multiple resistance genes in one of our isolates. The presence of clinically relevant antimicrobial resistance genes in

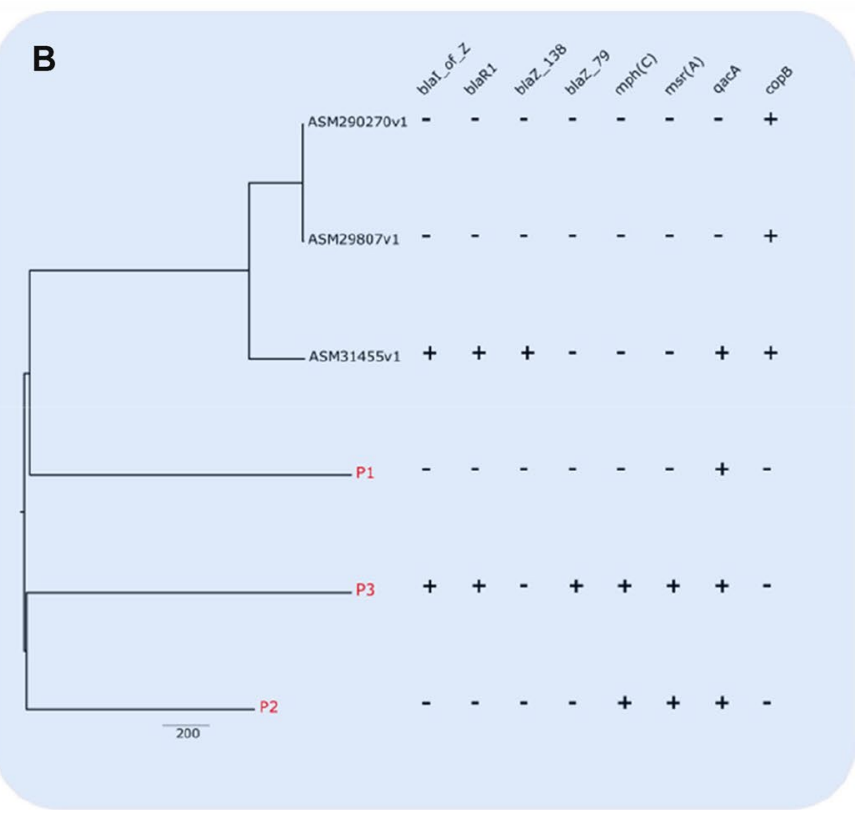

hemolyticus, and S. massiliensis). B Phylogenetic tree based on the core genome of $S$. massiliesis $(n=6)$ from our study and those previously published. Presence of antimicrobial resistance genes was assessed using the RESfinder database

skin commensals may pose therapeutic challenges and may be relevant for the acquisition of antibiotic resistances in facultative pathogens [18], such as members of the S. aureus complex, which warrants further investigations. 
In conclusion, our data add to the scarce body of evidence that $S$. massiliensis may be detected in clinical samples for routine microbiological diagnostics. Thus far, the clinical relevance is still unclear and further clinical and experimental studies are needed to elucidate the pathogenic properties and virulence of $S$. massiliensis. The technical advancement in microbiological diagnostics will introduce new depths in species identification and specification of novel organisms within the next years.

Funding Open Access funding enabled and organized by Projekt DEAL. This study has been funded by a 2021 CAREer Grant from the European Society of Clinical Microbiology and Infectious Diseases (ESCMID) to CP.

Data availability All relevant data are published within the article. Genome sequences of the 3 isolates investigated are available at the NCBI GenBank under project number PRJNA750592.

Code availability Not applicable.

\section{Declarations}

Ethics approval This study was approved by the local ethics committee (Number 147/21).

Consent to participate Due to the retrospective nature of the study and the anonymization of patient data, the need for informed consents was waived.

Consent for publication Not applicable.

Conflict of interest The authors declare no competing interests.

Open Access This article is licensed under a Creative Commons Attribution 4.0 International License, which permits use, sharing, adaptation, distribution and reproduction in any medium or format, as long as you give appropriate credit to the original author(s) and the source, provide a link to the Creative Commons licence, and indicate if changes were made. The images or other third party material in this article are included in the article's Creative Commons licence, unless indicated otherwise in a credit line to the material. If material is not included in the article's Creative Commons licence and your intended use is not permitted by statutory regulation or exceeds the permitted use, you will need to obtain permission directly from the copyright holder. To view a copy of this licence, visit http://creativecommons.org/licenses/by/4.0/.

\section{References}

1. Heilmann C, Ziebuhr W, Becker K (2019) Are coagulase-negative staphylococci virulent? Clin Microbiol Infect 25(9):1071-1080. https://doi.org/10.1016/j.cmi.2018.11.012

2. Michels R, Last K, Becker SL, Papan C (2021) Update on coagulase-negative staphylococci-what the clinician should know. Microorganisms 9(4):830. https://doi.org/10.3390/microorgan isms 9040830 
3. Nurjadi D, Last K, Klein S, Boutin S, Schmack B, Mueller F, Heeg K, Ruhparwar A, Heininger A, Zanger P (2020) Nasal colonization with Staphylococcus aureus is a risk factor for ventricular assist device infection in the first year after implantation: a prospective, single-centre, cohort study. J Infect 80(5):511-518. https://doi.org/10.1016/j.jinf.2020.02.015

4. Alhussein F, Fürstenberg J, Gaupp R, Eisenbeis J, Last K, Becker SL, Papan C (2020) Human infections caused by Staphylococcus argenteus in Germany: genetic characterisation and clinical implications of novel species designation. Eur J Clin Microbiol Infect Dis 39(12):2461-2465. https://doi.org/10.1007/ s10096-020-03950-4

5. Papan C, Karremann M, Weis M, Petzold A, Zahn K, Schroten H, Weichert S, Tenenbaum T (2021) A 28-day-old boy with multifocal osteomyelitis mimicking non-accidental injury. Klin Padiatr 233(2):91-93. https://doi.org/10.1055/a-1219-8053

6. Becker K, Both A, Weißelberg S, Heilmann C, Rohde H (2020) Emergence of coagulase-negative staphylococci. Expert Rev Anti Infect Ther 18(4):349-366. https://doi.org/10.1080/14787210. 2020.1730813

7. Papan C, Schroder M, Hoffmann M, Knoll H, Last K, Albrecht F, Geisel J, Fink T, Gartner BC, Mellmann A, Volk T, Berger FK, Becker SL (2021) Combined antibiotic stewardship and infection control measures to contain the spread of linezolid-resistant Staphylococcus epidermidis in an intensive care unit. Antimicrob Resist Infect Control 10(1):99. https://doi.org/10.1186/ s13756-021-00970-3

8. Pain M, Wolden R, Jaen-Luchoro D, Salva-Serra F, Iglesias BP, Karlsson R, Klingenberg C, Cavanagh JP (2020) Staphylococcus borealis sp. nov., isolated from human skin and blood. Int J Syst Evol Microbiol 70(12):6067-78. https://doi.org/10.1099/ijsem.0. 004499

9. Dupont C, Sivadon-Tardy V, Bille E, Dauphin B, Beretti JL, Alvarez AS, Degand N, Ferroni A, Rottman M, Herrmann JL, Nassif X, Ronco E, Carbonnelle E (2010) Identification of clinical coagulase-negative staphylococci, isolated in microbiology laboratories, by matrix-assisted laser desorption/ionization-time of flight mass spectrometry and two automated systems. Clin Microbiol Infect 16(7):998-1004. https://doi.org/10.1111/j.1469-0691.2009. 03036.x

10. De Bel A, Van Hoorde K, Wybo I, Vandoorslaer K, Echahidi F, De Brandt E, Schumann P, Ieven M, Soetens O, Piérard D, Vandamme P (2013) Staphylococcus jettensis sp. nov., a coagulase-negative staphylococcal species isolated from human clinical specimens. Int J Syst Evol Microbiol. 63(Pt 9):3250-6. https://doi.org/10. 1099/ijs.0.044438-0

11 Al Masalma M, Raoult D, Roux V (2010) Staphylococcus massiliensis sp. nov., isolated from a human brain abscess. Int J Syst Evol Microbiol. 60(Pt 5):1066-72. https://doi.org/10.1099/ijs.0. 006486-0

12. Srivastav R, Singh A, Jangir PK, Kumari C, Muduli S, Sharma R (2013) Genome sequence of Staphylococcus massiliensis strain S46, isolated from the surface of healthy human skin. Genome Announc 1(4):e00553-13. https://doi.org/10.1128/genomeA. 00553-13

13. Zong Z (2012) The newly-recognized species Staphylococcus massiliensis is likely to be part of the human skin microflora. Antonie Van Leeuwenhoek 101(2):449-451. https://doi.org/10. 1007/s10482-011-9635-5

14. Klein S, Hannesen J, Zanger P, Heeg K, Boutin S, Nurjadi D (2020) Entry of Panton-Valentine leukocidin-positive methicillinresistant Staphylococcus aureus into the hospital: prevalence and population structure in Heidelberg, Germany 2015-2018. Sci Rep 10(1):13243. https://doi.org/10.1038/s41598-020-70112-z

15. Richter M, Rosselló-Móra R (2009) Shifting the genomic gold standard for the prokaryotic species definition. Proc Natl Acad Sci U S A 106(45):19126-19131. https://doi.org/10.1073/pnas. 0906412106

16. Laurent F, Butin M (2019) Staphylococcus capitis and NRCS-A clone: the story of an unrecognized pathogen in neonatal intensive care units. Clin Microbiol Infect 25(9):1081-1085. https://doi.org/ 10.1016/j.cmi.2019.03.009

17. Zong Z, Peng C, Lü X (2011) Diversity of SCCmec elements in methicillin-resistant coagulase-negative staphylococci clinical isolates. PLoS ONE 6(5):e20191. https://doi.org/10.1371/journal. pone.0020191

18. Morley VJ, Woods RJ, Read AF (2019) Bystander selection for antimicrobial resistance: implications for patient health. Trends Microbiol 27(10):864-877. https://doi.org/10.1016/j.tim.2019.06. 004

Publisher's note Springer Nature remains neutral with regard to jurisdictional claims in published maps and institutional affiliations. 